\title{
Outcomes of Patients With Cancer Discharged to a Skilled Nursing Facility After Acute Care Hospitalization
}

\author{
Sarguni Singh, MD ${ }^{1}$; Megan Eguchi, $\mathrm{MPH}^{2}$; Sung-Joon Min, $\mathrm{PhD}^{3}$; and Stacy Fischer, $\mathrm{MD}^{4}$
}

\section{ABSTRACT}

Background: After discharge from an acute care hospitalization, patients with cancer may choose to pursue rehabilitative care in a skilled nursing facility (SNF). The objective of this study was to examine receipt of anticancer therapy, death, readmission, and hospice use among patients with cancer who discharge to an SNF compared with those who are functionally able to discharge to home or home with home healthcare in the 6 months after an acute care hospitalization. Methods: A population-based cohort study was conducted using the SEER-Medicare database of patients with stage II-IV colorectal, pancreatic, bladder, or lung cancer who had an acute care hospitalization between 2010 and 2013. A total of 58,770 cases were identified and patient groups of interest were compared descriptively using means and standard deviations for continuous variables and frequencies and percentages for categorical variables. Logistic regression was used to compare patient groups, adjusting for covariates. Results: Of patients discharged to an SNF, 21\%, 17\%, and $2 \%$ went on to receive chemotherapy, radiotherapy, and targeted chemotherapy, respectively, compared with $54 \%, 28 \%$, and $6 \%$, respectively, among patients discharged home. Fifty-six percent of patients discharged to an SNF died within 6 months of their hospitalization compared with $36 \%$ discharged home. Thirty-day readmission rates were $29 \%$ and $28 \%$ for patients discharged to an SNF and home, respectively, and $12 \%$ of patients in hospice received $<3$ days of hospice care before death regardless of their discharge location. Conclusions: Patients with cancer who discharge to an SNF are significantly less likely to receive subsequent oncologic treatment of any kind and have higher mortality compared with patients who discharge to home after an acute care hospitalization. Further research is needed to understand and address patient goals of care before discharge to an SNF.

J Natl Compr Canc Netw 2020;18(7):856-865 doi: 10.6004/jnccn.2020.7534

\footnotetext{
${ }^{1}$ Division of Hospital Medicine, University of Colorado Denver; ${ }^{2}$ University of Colorado Cancer Center; ${ }^{3}$ Division of Health Care Policy \& Research, and ${ }^{4}$ Division of General Internal Medicine, University of Colorado Denver, Aurora, Colorado.
}

\section{Background}

Studies have shown a significant correlation between functional status and survival for patients with cancer. ${ }^{1-3}$ The functional decline associated with progressive cancer is prognostic for health events that severely limit mobility and also with acute care hospitalizations. ${ }^{4}$ After discharge from an acute care hospitalization, patients may choose to pursue rehabilitative care in a skilled nursing facility (SNF). SNF care is considered a transitional period during which patients who are too weak to discharge home can receive skilled nursing care and rehabilitative therapies. Patients who discharge to an SNF are typically older, more medically complex, and have higher hospital readmission rates than those who are strong enough to discharge home. ${ }^{5-7}$

Use of institutional postacute care increased from $21 \%$ in 2000 to $26 \%$ in 2015, resulting in Medicare spending $>\$ 59$ billion for these services in $2015 .{ }^{8,9} \mathrm{SNF}$ services are covered by Medicare Part A (hospital insurance), which provides payments to SNFs for a set period of time to care for patients after a qualifying hospital stay. Importantly, the SNF is the only setting that Medicare reimburses for 24-hour care after an acute care hospitalization for patients who are not eligible for longterm acute care or inpatient rehabilitation.

There are 2 important limitations for patients with cancer who discharge to an SNF. First, patients with cancer rarely receive chemotherapy while admitted to an SNF because infusion chemotherapy is considered an outpatient procedure and covered by Medicare Part B whereas care in SNFs is covered by Part A. Therefore, to provide chemotherapy, SNFs would need to absorb the cost of administration and treatment. Second, availability of palliative care consultation remains limited for patients in SNFs. ${ }^{10}$

We sought to understand clinical outcomes of patients with stage II-IV pancreatic, colorectal, lung, and bladder cancers who discharge to an SNF compared with

See JNCCN.org for supplemental online content. 
patients who discharge to home or home with home healthcare. These cancer types were selected because we anticipated that these patients would have high rates of hospital admission and readmission and would likely be candidates for SNF discharge, thus providing a robust cohort of patients for analysis. We assessed healthcare utilization by examining rates of receipt of cancerdirected therapy, 30-day readmission, death, and hospice use 6 months after an acute care hospitalization. We hypothesized that most patients who are discharged to an SNF do not subsequently receive oncologic treatment, have higher mortality and readmission rates, and have lower hospice use compared with patients who discharge to home or home with home healthcare.

\section{Methods}

\section{Data Source}

The data source was the SEER-Medicare database. The SEER program collects data from select cancer registries covering approximately $28 \%$ of the US population; $93 \%$ of persons aged $\geq 65$ years in the SEER files are matched to the Medicare enrollment file. During our study, $72 \%$ to $76 \%$ of the Medicare population enrolled in Medicare Fee-for-Service (FFS). For cases enrolled in Medicare FFS, the combined file provides detailed demographic and clinical information collected by cancer registries at diagnosis plus covered healthcare services received before diagnosis, during initial treatment, and over the course of follow-up for the duration of available claims. The database also includes a limited set of census tract and zip code-level socioeconomic variables. ${ }^{11}$

\section{Sample Selection}

We selected patients with colorectal, pancreatic, bladder, or lung cancer diagnosed between 2010 and 2013. We excluded records from patients with a subsequent primary tumor or other prior cancer diagnoses besides stage 0 or I breast or cervical cancer, or nonmetastatic prostate cancer diagnosed in the 3 years before the tumor of interest (total sample size: $n=301,776$, including 5,242 patients with one of the accepted prior tumors specified). Our analysis only included de novo cancers because information about recurrent cancers is not provided in the SEER-Medicare database. We further restricted to patients with AJCC 7th edition stage group II-IV tumors at diagnosis to obtain the patient sample with regional or advanced disease $(\mathrm{n}=190,692){ }^{12,13}$

We identified the patient sample for which we had complete claims data by restricting it to patients aged $\geq 66$ years at diagnosis $(n=150,679)$ and excluding those diagnosed at autopsy and those with a missing diagnosis date $(n=127)$ or with negative survival time $(\mathrm{n}=359)$. We included only patients who were continuously enrolled in Medicare FFS Parts A and B from 12 months before diagnosis through death or the end of the study follow-up, December 2014 ( $n=91,568)$.

The study sample was further limited to patients with a paid claim for a short-term inpatient stay subsequent to diagnosis that did not end in death or discharge to hospice $(n=64,160)$. We assigned the first stay occurring in the month of diagnosis or later as the index inpatient stay. We required that the index stay occur by June 2014, with continuous enrollment in Medicare FFS Parts A and B for at least 6 months after discharge or until death if before 6 months, to ensure adequate follow-up for all outcome measures $(n=63,697)$. Furthermore, removing discharge locations other than the 3 settings of interest (eg, inpatient residential facility, Medicarecertified long-term care hospital, transfer to another hospital) brought the total sample to 58,770 patients. Of these, $29 \%(n=16,936)$ had a prior oncologic visit (established) and $71 \%(n=41,834)$ had no prior oncologic visit (unestablished) (Figure 1). The Colorado Multiple Institutional Review Board and Duke Institutional Review Board approved this study.

\section{Comparator Groups}

Analyses were conducted among 3 comparison groups with discharge locations of interest: SNF, home, and home with home healthcare $(n=58,770)$. We defined a confirmed discharge to an SNF as the presence of an SNF claim with an admission date equal to the index discharge date. Discharges to home and to home with home healthcare were identified using the patient discharge status code reported on the inpatient claim.

\section{Outcomes}

The primary outcome was receipt of anticancer therapy after an inpatient hospitalization in the 6 months after discharge. CPT codes, the Healthcare Common Procedure Coding System (HCPCS) codes, ICD-9 procedure and diagnosis codes, and National Drug Codes (NDCs) were used to identify treatment received, including radiotherapy (RT), chemotherapy, and targeted therapy (see supplemental eAppendix 1, available with this article at JNCCN.org). We included targeted therapy drugs approved by the FDA for the tumor sites of interest that had specific HCPCS codes initiated before December 2014. The selected drugs included bevacizumab, cetuximab, everolimus, panitumumab, ramucirumab, and ziv-aflibercept. Claims in the 6 months after discharge were used to obtain additional outcome measures: 30-day readmission, hospice use, and death. Hospice use was measured using the number of covered days of care reported on hospice claims in the 6 months after index discharge. 


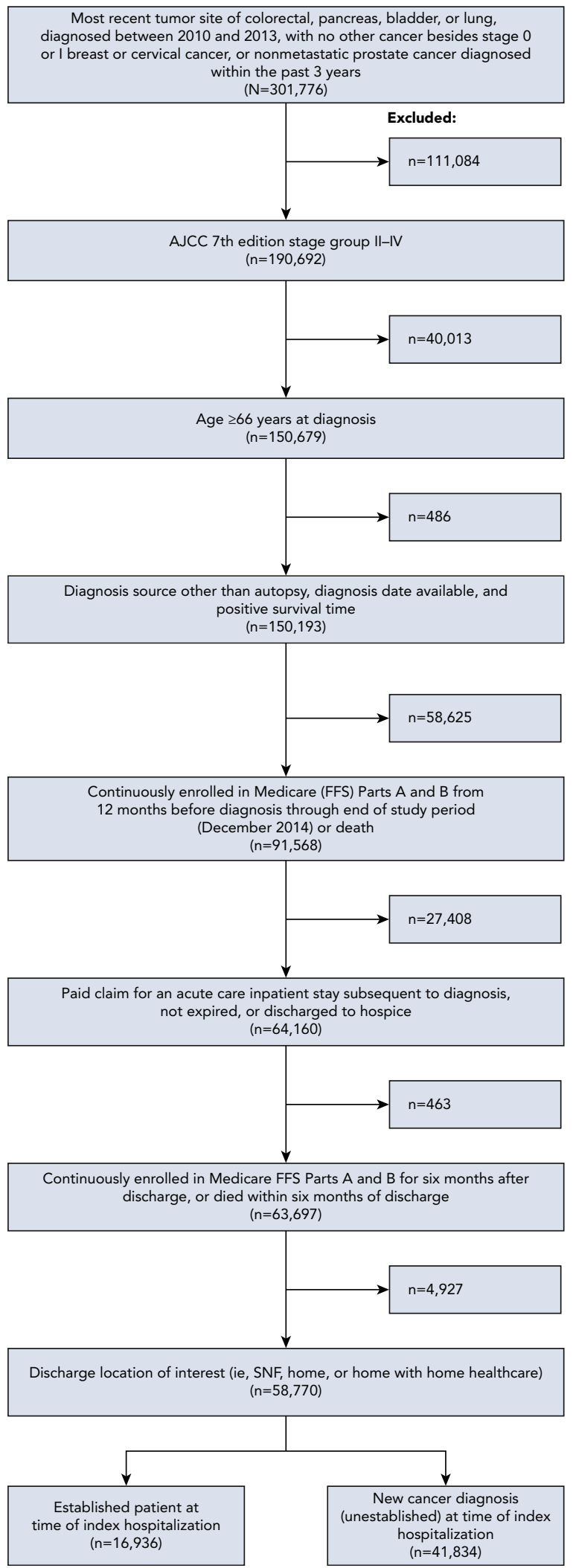

Figure 1. SEER-Medicare analysis sample derivation. Abbreviations: FFS, fee-for-service; SNF, skilled nursing facility.

\section{Control Variables}

We used SEER variables to obtain patient demographics and tumor characteristics at diagnosis, and used claims to identify characteristics of the index inpatient stay, prior health conditions, and healthcare services received, and to generate the Charlson comorbidity index and identify specific conditions of interest using Centers for Medicare and Medicaid Services' Chronic Conditions Data Warehouse algorithms. We also used treatment and other healthcare services received after diagnosis and before index admission, including visits with an oncology specialist, prior RT, chemotherapy, and targeted therapy, as covariates in the analysis. ${ }^{14}$

\section{Statistical Analysis}

The 3 patient groups of interest were compared descriptively using means and standard deviations for continuous variables and frequencies and percentages for categorical variables. SEER-Medicare provides only the month of the cancer diagnosis but does not include date or setting in which the diagnosis was made. Patients with and without prior oncologist visits were considered separately in subsequent analyses to identify those patients with a known cancer diagnosis before index hospitalization versus those diagnosed with a new cancer at the time of index hospitalization. Subsequent cancerdirected treatment, mortality, readmission, and hospice use were compared by discharge setting using $\chi^{2}$ tests. Logistic regression was used to compare patient groups, adjusting for covariates. Kaplan-Meier estimates were used to compare survival curves by patient group. For patients discharged to SNF, statistically significant predictors for the outcomes were identified using logistic regression and Cox proportional hazards regression. The regression models were developed using a random $50 \%$ subsample and validated on the remaining $50 \%$ subsample. Statistical significance was defined as $P<.05$.

\section{Results}

\section{Patient Characteristics}

The study population consisted of 58,770 people with stage II-IV colorectal (31\%), lung (51\%, with $77 \%$ nonsmall cell lung cancer), pancreatic $(12 \%)$, or bladder cancer $(6 \%)$ (Table 1). A total of $71 \%$ of patients were given a new cancer diagnosis at the time of index hospitalization (unestablished patients), whereas $29 \%$ had a known cancer diagnosis before index hospitalization (established patients). Mean (SD) length of stay (LOS) for the index hospitalization was $6.9( \pm 5.8)$ days for all patients. LOS was $5.0( \pm 3.8)$ days for patients discharging to home, $8.1( \pm 5.8)$ days for those discharging to home with home healthcare, and $10.6( \pm 7.6)$ days for those discharging to an SNF. 
Table 1. Patient and Index Hospitalization Characteristics by Discharge Setting

\begin{tabular}{|c|c|c|c|c|}
\hline Characteristic & $\begin{array}{c}\text { All } \\
\text { n (\%) }\end{array}$ & $\begin{array}{l}\text { Home } \\
\text { n (\%) }\end{array}$ & $\begin{array}{l}\text { Home Healthcare } \\
\text { n (\%) }\end{array}$ & $\begin{array}{l}\text { SNF } \\
\text { n (\%) }\end{array}$ \\
\hline Patients & $58,770(100)$ & $33,057(56)$ & $13,592(23)$ & $12,121(21)$ \\
\hline \multicolumn{5}{|l|}{ Median age (SD), y } \\
\hline Index admission & $77.6( \pm 7.1)$ & $76.2( \pm 6.6)$ & $77.9( \pm 7.0)$ & $81.0( \pm 7.2)$ \\
\hline White non-Hispanic & $47,668(81.2)$ & $26,733(81.0)$ & $10,826(79.7)$ & $10,109(83.4)$ \\
\hline Black non-Hispanic & $4,974(8.5)$ & $2,697(8.2)$ & $1,331(9.8)$ & $946(7.8)$ \\
\hline Hispanic & $3,070(5.2)$ & $1,791(5.4)$ & $746(5.5)$ & $533(4.4)$ \\
\hline $\begin{array}{l}\text { Asian or Pacific Islander/American Indian/Alaska } \\
\text { Native non-Hispanic }\end{array}$ & $3,002(5.1)$ & $1,803(5.5)$ & $678(5.0)$ & $521(4.3)$ \\
\hline \multicolumn{5}{|l|}{ Census tract SES } \\
\hline$\%$ of residents below poverty level (SD) & $14.0( \pm 9.3)$ & $14.3( \pm 9.3)$ & $14.1( \pm 9.3)$ & $13.2( \pm 9.1)$ \\
\hline$\%$ of non-high school graduates (SD) & $14.4( \pm 9.8)$ & $14.6( \pm 9.9)$ & $14.6( \pm 10.1)$ & $13.7( \pm 9.5)$ \\
\hline \multicolumn{5}{|l|}{ Charlson comorbidity score } \\
\hline 0 & $19,452(33.1)$ & $11,848(35.8)$ & $4,210(30.1)$ & $3,394(28.0)$ \\
\hline 1 & $16,946(28.8)$ & $9,879(29.9)$ & $3,933(28.9)$ & $3,134(25.9)$ \\
\hline 2 & $9,845(16.8)$ & $5,288(16.0)$ & 2,333 (17.2) & $2,224(18.4)$ \\
\hline$\geq 3$ & $12,527(21.3)$ & $6,042(18.3)$ & $3,116(22.9)$ & $3,369(27.8)$ \\
\hline \multicolumn{5}{|l|}{ Year of diagnosis } \\
\hline 2010 & $15,334(26.1)$ & $8,601(26.0)$ & $3,574(26.3)$ & $3,159(26.1)$ \\
\hline \multicolumn{5}{|l|}{ Cancer type/stage } \\
\hline Stage III & $6,528(35.3)$ & 3,545 (36.7) & $1,474(34.8)$ & $1,509(33.0)$ \\
\hline Stage IV & $4,446(24.1)$ & $2,180(22.6)$ & $1,090(25.7)$ & $1,176(25.8)$ \\
\hline Lung & $29,792(50.7)$ & $17,218(52.1)$ & $6,823(50.2)$ & $5,751(47.5)$ \\
\hline Stage II & $3,551(11.9)$ & $2,139(12.4)$ & $834(12.2)$ & $578(10.1)$ \\
\hline Stage III & $7,634(25.6)$ & $4,688(27.2)$ & $1,655(24.3)$ & $1,291(22.5)$ \\
\hline Stage IV & $18,607(62.5)$ & $10,391(60.4)$ & $4,334(63.5)$ & $3,882(67.5)$ \\
\hline Pancreatic & 6,897 (11.7) & $4,303(13.0)$ & $1,531(11.3)$ & $1,063(8.8)$ \\
\hline Stage II & $2,521(36.6)$ & $1,507(35.0)$ & $613(40.0)$ & $401(37.7)$ \\
\hline Stage III & $692(10.0)$ & 469 (10.9) & $135(8.8)$ & $88(8.3)$ \\
\hline Stage IV & $3,684(53.4)$ & $2,327(54.1)$ & $783(51.1)$ & $574(54.0)$ \\
\hline Bladder & $3,609(6.1)$ & $1,870(5.7)$ & 999 (7.4) & $740(6.1)$ \\
\hline Stage II & $2,000(55.4)$ & $1,037(55.5)$ & $531(53.2)$ & $432(58.4)$ \\
\hline Stage III & $639(17.7)$ & 335 (17.9) & $196(19.6)$ & $108(14.6)$ \\
\hline Stage IV & $970(26.9)$ & $498(26.6)$ & $272(27.2)$ & $200(27.0)$ \\
\hline
\end{tabular}




\begin{tabular}{|c|c|c|c|c|}
\hline Characteristic & $\begin{array}{c}\text { All } \\
\text { n (\%) }\end{array}$ & $\begin{array}{l}\text { Home } \\
\text { n (\%) }\end{array}$ & $\begin{array}{l}\text { Home Healthcare } \\
\text { n (\%) }\end{array}$ & $\begin{array}{l}\text { SNF } \\
\text { n (\%) }\end{array}$ \\
\hline \multicolumn{5}{|l|}{ Healthcare utilization before index admission } \\
\hline$\geq 1$ SNF stays & $5,181(8.8)$ & $1,279(3.9)$ & $800(5.9)$ & $3,102(25.6)$ \\
\hline Visit with oncologist & $16,936(28.8)$ & $9,763(29.5)$ & $4,351(32.0)$ & $2,822(23.3)$ \\
\hline Chemotherapy & $9,584(16.3)$ & $5,588(16.9)$ & $2,529(18.6)$ & $1,467(12.1)$ \\
\hline Radiotherapy & $6,641(11.3)$ & $3,652(11.1)$ & $1,803(13.3)$ & $1,186(9.8)$ \\
\hline Targeted chemotherapy & $1,131(1.9)$ & $669(2.0)$ & $281(2.1)$ & $181(1.5)$ \\
\hline \multicolumn{5}{|l|}{ Index hospital characteristics } \\
\hline \multicolumn{5}{|l|}{ Location } \\
\hline Urban (vs rural) & $53,137(91.2)$ & $29,746(90.1)$ & $12,401(92.1)$ & $10,990(90.7)$ \\
\hline \multicolumn{5}{|l|}{ Type } \\
\hline Teaching (vs nonteaching) & $32,107(55.1)$ & $17,847(54.5)$ & $7,767(57.7)$ & $6,493(53.6)$ \\
\hline \multicolumn{5}{|l|}{ Index stay DRG (top 10 listed, *surgical) } \\
\hline $\begin{array}{l}\text { *330 Major small and large bowel procedures } \\
\text { with CC }\end{array}$ & $7,117(12.2)$ & $3,704(11.3)$ & $1,768(13.1)$ & $1,645(13.6)$ \\
\hline 181 Respiratory neoplasms with CC & $3,441(5.9)$ & $2,117(6.4)$ & $730(5.4)$ & $594(4.9)$ \\
\hline 180 Respiratory neoplasms with MCC & $3,196(5.5)$ & $1,563(4.7)$ & $840(6.2)$ & $793(6.6)$ \\
\hline $\begin{array}{l}\text { * } 329 \text { Major small and large bowel procedures } \\
\text { with MCC }\end{array}$ & $2,767(4.7)$ & $615(1.9)$ & $762(5.6)$ & $1,390(11.5)$ \\
\hline $\begin{array}{l}\text { *331 Major small and large bowel procedures } \\
\text { without CC/MCC }\end{array}$ & $2,477(4.2)$ & $1,813(5.5)$ & $428(3.2)$ & $236(2.0)$ \\
\hline *164 Major chest procedures with CC & $2,276(3.9)$ & $1,399(4.3)$ & $623(4.6)$ & $254(2.1)$ \\
\hline $\begin{array}{l}\text { * } 167 \text { Other respiratory system OR procedures } \\
\text { with CC }\end{array}$ & $1,437(2.5)$ & $895(2.7)$ & $350(2.6)$ & $192(1.6)$ \\
\hline $\begin{array}{l}\text { * } 166 \text { Other respiratory system OR procedures } \\
\text { with MCC }\end{array}$ & $1,315(2.2)$ & $618(1.9)$ & $367(2.7)$ & $330(2.7)$ \\
\hline $\begin{array}{l}435 \text { Malignancy of hepatobiliary system or } \\
\text { pancreas with MCC }\end{array}$ & $1,276(2.2)$ & $798(2.4)$ & $283(2.1)$ & $195(1.6)$ \\
\hline 375 Digestive malignancy with CC & $1,258(2.1)$ & $898(2.7)$ & $180(1.3)$ & $180(1.5)$ \\
\hline
\end{tabular}

Data were rarely missing $(<1 \%$, except SES variables $\sim 4 \%)$. $P$ values are not shown because all comparisons were highly significant due to the large sample size. Abbreviations: CC, complication or comorbidity; DRG, diagnosis-related group; MCC, major complication or comorbidity; OR, operating room; SES, socioeconomic status; SNF, skilled nursing facility.

\section{Patient Outcomes}

Of SNF discharges, $21 \%, 17 \%$, and $2 \%$ went on to receive chemotherapy, RT, and targeted chemotherapy, respectively, compared with $54 \%, 28 \%$, and $6 \%$, respectively, among home discharges. Furthermore, 56\% of SNF discharges died within 6 months of hospitalization compared with $36 \%$ of patients who discharged home (Figure 2A, B). Thirty-day readmission rates were $29 \%$ and $28 \%$ for SNF and home discharges, respectively, and $12 \%$ of patients in hospice received $<3$ days of hospice care before their death regardless of their discharge location. Patients who had a major bowel surgery seemed more likely to require SNF care after hospitalization.

A total of $29 \%$ of unestablished patients who discharged to an SNF went on to receive any cancer treatment (chemotherapy, RT, targeted chemotherapy) compared with $60 \%$ of patients discharged home (Table 2).
For patients with a known cancer diagnosis and receipt of prior treatment, $42 \%$ of those discharged to an SNF went on to receive any further cancer treatment compared with $74 \%$ of those discharged home (Table 3 ).

\section{Predictors of SNF Success}

For unestablished patients, those with lung, pancreatic, and bladder cancer were more likely to receive any further treatment compared with those with colorectal cancer. Unestablished patients with stage III cancer were more likely to receive future treatment compared with those with stage IV cancer. Both established and unestablished patients with stage II cancer were less likely to receive future oncologic treatment compared with those with stage IV cancer. Both established and unestablished patients with cognitive impairment were less likely to receive future treatment. 
A

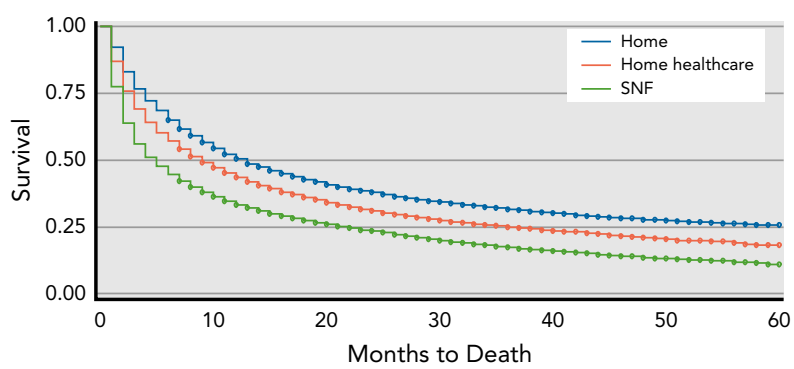

B

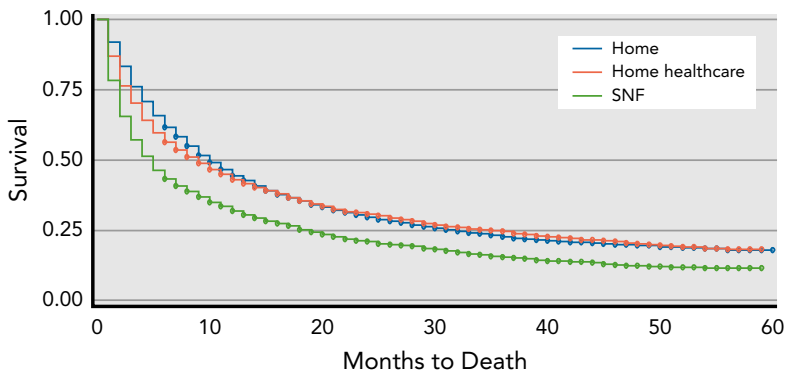

Figure 2. Kaplan-Meier survival curves for patients with (A) unestablished and (B) established cancer. Abbreviation: SNF, skilled nursing facility.

For all patients discharged to an SNF, stage II and III cancer was associated with a lower risk of death and longer time to death (measured in months), indicated by a lower hazard of death compared with stage IV cancer. For established patients with cancer, those who had received chemotherapy before their index hospitalization were less likely to die compared with those who had received no treatment before index admission (Table 4).

\section{Discussion}

This study describes postacute care outcomes of patients with stage II-IV colorectal, lung, pancreatic, and bladder cancer discharged to an SNF after an acute care hospitalization. Data show that these patients are significantly less likely to go on and receive cancer treatment of any kind and are more likely to die within 6 months of discharge compared with patients discharged home. Only approximately one-fifth of patients discharged to an SNF received subsequent chemotherapy compared with slightly more than half of patients discharged home. This analysis also shows how ill this cancer population is: $42 \%$ of all patients who had an acute care hospitalization who were discharged to home, home with healthcare services, or an SNF had died within 6 months of discharge.

Although these findings are not unexpected, the magnitude of difference in outcomes of patients who

Table 2. Outcomes at 6 Months for Patients With Unestablished Cancer $(\mathrm{N}=41,834)$

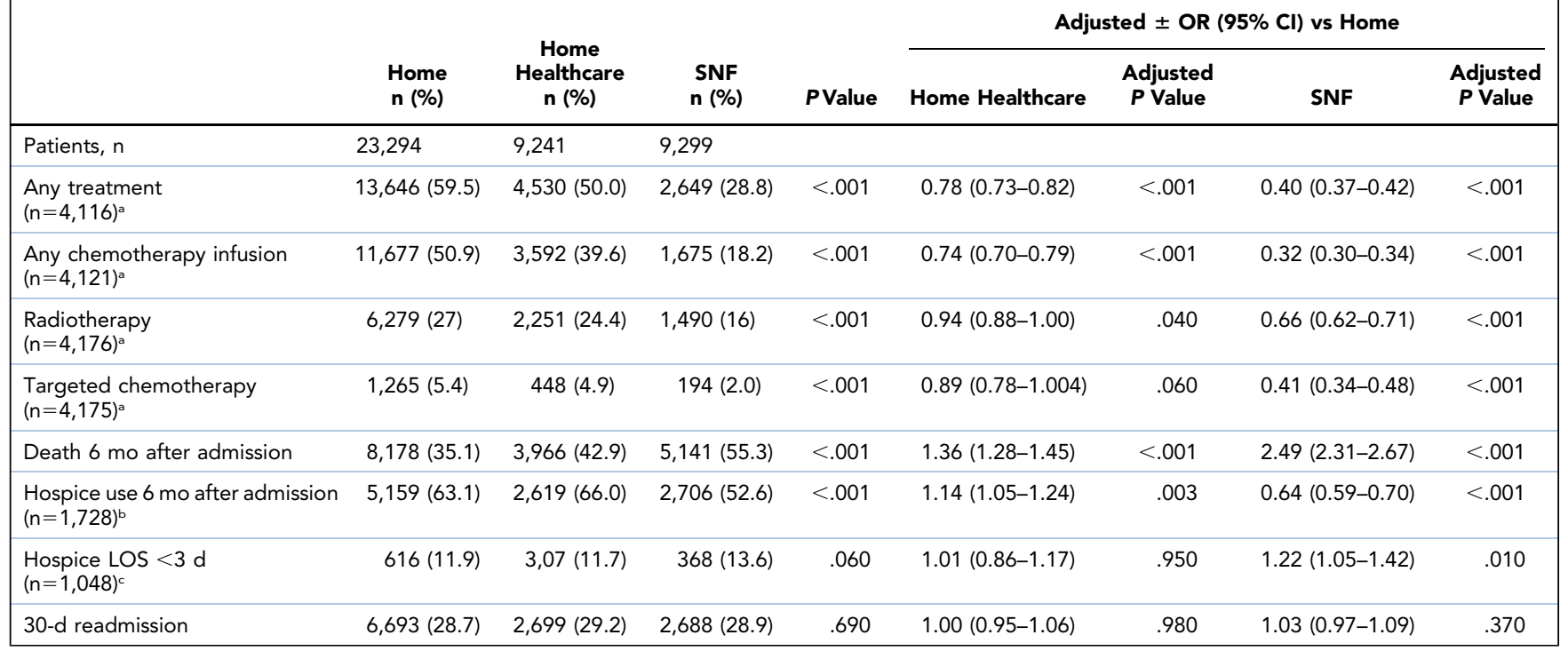

\pm prior treatment (prior chemotherapy, prior radiotherapy, and prior targeted chemotherapy; or prior any therapy for any treatment outcome), discharge year, cancer type and stage, histologic grade, patient age at index admission, months between diagnosis and index admission, male sex, white non-Hispanic race, urban location, index hospital region and characteristics, prior hypertension, prior diabetes, prior chronic obstructive pulmonary disease, prior heart disease, prior stroke, prior cognitive disorder, index admission LOS, Charlson comorbidity index categories, per capita income, median income, percent of non-high school graduates, percent of residents below poverty level, married or partnered relationship status, prior depressive disorders, prior alcohol use disorder, prior drug use, and prior tobacco use. Abbreviations: LOS, length of stay; OR, odds ratio; SNF, skilled nursing facility.

aExcludes few cases with data error where prior cancer treatment is indicated.

bPatients who died.

'Patients who received hospice before death. 
Table 3. Outcomes at 6 Months for Patients With Established Cancer $(\mathrm{N}=16,936)$

\begin{tabular}{|c|c|c|c|c|c|c|c|c|}
\hline & \multirow[b]{2}{*}{$\begin{array}{l}\text { Homen } \\
(\%)\end{array}$} & \multirow{2}{*}{$\begin{array}{c}\text { Home } \\
\text { Healthcare } \\
\text { n (\%) }\end{array}$} & \multirow[b]{2}{*}{$\begin{array}{l}\text { SNF } \\
\text { n (\%) }\end{array}$} & \multirow[b]{2}{*}{$\begin{array}{c}P \\
\text { Value }\end{array}$} & \multicolumn{4}{|c|}{ Adjusted $\pm \mathrm{OR}(95 \% \mathrm{Cl})$ vs Home } \\
\hline & & & & & $\begin{array}{c}\text { Home } \\
\text { Healthcare }\end{array}$ & $\begin{array}{l}\text { Adjusted } \\
P \text { Value }\end{array}$ & SNF & $\begin{array}{c}\text { Adjusted } \\
P \text { Value }\end{array}$ \\
\hline Any treatment & & & & & $0.72(0.67-0.79)$ & $<.001$ & $0.39(0.35-0.43)$ & $<.001$ \\
\hline Prior receipt $(n=10,648)^{a}$ & $4,483(73.5)$ & $1,772(63.3)$ & $736(42.1)$ & $<.001$ & & & & \\
\hline Radiotherapy & & & & & $0.83(0.76-0.91)$ & $<.001$ & $0.66(0.59-0.75)$ & $<.001$ \\
\hline Prior receipt $(n=6,570)^{a}$ & $1,496(41.5)$ & $553(30.9)$ & $299(25.4)$ & $<.001$ & & & & \\
\hline Targeted chemotherapy & & & & & $0.71(0.58-0.85)$ & $<.001$ & $0.44(0.33-0.57)$ & $<.001$ \\
\hline Prior receipt $(n=1,054)^{a}$ & $287(45.6)$ & $93(35.6)$ & $36(22.1)$ & $<.001$ & & & & \\
\hline 30-d readmission & $2,403(24.6)$ & $1,245(28.6)$ & $823(29.2)$ & $<.001$ & $1.20(1.10-1.31)$ & $<.001$ & $1.20(1.07-1.34)$ & .001 \\
\hline
\end{tabular}

\pm prior treatment (prior chemotherapy, prior radiotherapy, and prior targeted chemotherapy; or prior any therapy for any treatment outcome), discharge year, cancer type and stage, histologic grade, patient age at index admission, months between diagnosis and index admission, male sex, white non-Hispanic race, urban location, index hospital region and characteristics, prior hypertension, prior diabetes, prior chronic obstructive pulmonary disease, prior heart disease, prior stroke, prior cognitive disorder, index admission LOS, Charlson comorbidity index categories, per capita income, median income, percent of non-high school graduates, percent of residents below poverty, married or partnered relationship status, prior depressive disorders, prior alcohol use disorder, prior drug use, and prior tobacco use. Abbreviations: LOS, length of stay; OR, odds ratio; SNF, skilled nursing facility.

aPatients who received cancer treatment before index hospitalization.

bPatients who died.

cPatients who received hospice before death.

discharge to an SNF compared with those who discharge home is striking. This analysis of SEER-Medicare data confirms previous research of SNF populations in general. ${ }^{5-7}$ Patients who discharge to an SNF are more frail, older, and have more comorbidities than those who discharge home. Thus, poorer outcomes in the SNF population of this study are not unexpected but rather most congruent with their projected outcomes based on their clinical morbidities. Although we attempted to adjust for patient-level differences, including hospital characteristics, the cohorts are inherently different, and the discharge location essentially serves as a surrogate for functional status. The intent of this study was to understand the impact of discharge location, as a surrogate for function, on subsequent rates of cancer-directed treatment. ASCO recommends against the use of chemotherapy in patients with solid tumors who have not benefited from prior treatment and who have an ECOG ${ }^{15}$ performance status (PS) score $\geq 3 .{ }^{16}$ These findings suggest that if the intent of sending this patient population to an SNF is to recover functional status and receive cancerdirected therapy, most patients will not realize this goal.

Discharging patients with cancer who have a poor prognosis to an SNF setting hinders their ability to express their goals of care and to participate in end-of-life planning because of the limited access to palliative care in SNFs. Previous studies have shown that oncologists struggle with communicating prognosis and with saying "no" to continued chemotherapy treatment in patients with end-stage cancer. ${ }^{17,18}$ This struggle likely contributes to the poor prognostic understanding and unrealistic expectations patients experience at the end of life. The proportion of patients using short-term hospice services ( $\leq 3$ days) decreased from $9.8 \%$ in 2009 to $7.7 \%$ in $2015 .^{19}$ Our study revealed that $13.6 \%$ and $11.9 \%$ of patients who discharged to an SNF or home, respectively, had a hospice LOS $<3$ days. These numbers are higher than national averages for both discharge locations (SNF and home). It is possible that the standard Medicare requirement to select either hospice or SNF care may lead to lower hospice enrollment. The Medicare Care Choices Model, allowing for concurrent hospice and cancerdirected treatment, might increase hospice utilization in this population. ${ }^{20}$

This study reveals differences between patients with established cancer and those who were newly diagnosed with cancer. Patients with unestablished cancer were less likely to receive future oncologic treatment at all discharge locations compared with patients with established cancer who had received 


\section{Table 4. Predictors of SNF Success in Outcomes at 6 Months for Patients With Established Cancer}

\begin{tabular}{|c|c|c|c|c|c|c|}
\hline Variable & $\begin{array}{l}\text { Any Treatment } \\
\text { OR }(95 \% \mathrm{Cl})\end{array}$ & $\begin{array}{c}\text { Death } \\
\text { OR }(95 \% \mathrm{Cl})\end{array}$ & $\begin{array}{c}\text { Hospice } \\
\text { OR }(95 \% \mathrm{Cl})\end{array}$ & $\begin{array}{c}\text { Hospice } \\
\text { LOS <3 D } \\
\text { OR }(95 \% \mathrm{Cl})\end{array}$ & $\begin{array}{c}\text { 30-D } \\
\text { Readmission } \\
\text { OR }(95 \% \mathrm{Cl})\end{array}$ & $\begin{array}{l}\text { Months to } \\
\text { Death } \\
\text { HR }(95 \% \mathrm{Cl})\end{array}$ \\
\hline \multirow[t]{2}{*}{ Patients, $\mathrm{n}$} & 1,411 & 1,397 & 805 & 460 & 1,397 & 1,397 \\
\hline & $c=0.64$ & $c=0.75$ & $c=0.66$ & $c=0.57$ & $c=0.62$ & \\
\hline Male (vs female) & & $1.66(1.30-2.11)$ & & & & $1.22(1.08-1.37)$ \\
\hline Age at index admission & $0.96(0.94-0.98)$ & & & & & $1.02(1.01-1.02)$ \\
\hline $\begin{array}{l}\text { Months between diagnosis and } \\
\text { index admission }\end{array}$ & $0.95(0.93-0.97)$ & & & & & \\
\hline White non-Hispanic race (vs other) & & & $1.54(0.99-2.38)$ & & & \\
\hline \multicolumn{7}{|l|}{ Married or partnered (vs nonmarried) } \\
\hline Urban location & & & $1.85(1.15-2.98)$ & & & $1.27(1.00-1.61)$ \\
\hline \multicolumn{7}{|l|}{ SES } \\
\hline \multicolumn{7}{|l|}{ Per capita income ( $\$ 1,000$ USD) } \\
\hline \multicolumn{7}{|l|}{ Median income ( $\$ 1,000$ USD) } \\
\hline \multicolumn{7}{|l|}{$\%$ of residents below poverty level } \\
\hline \multicolumn{7}{|l|}{$\%$ of non-high school graduates } \\
\hline $\begin{array}{l}\text { Charlson comorbidity index } \\
\text { categories }\end{array}$ & & & & & $1.16(1.04-1.29)$ & \\
\hline \multicolumn{7}{|l|}{ Cancer type (vs colorectal) } \\
\hline Lung & & $2.83(2.05-3.92)$ & & & $1.32(0.97-1.79)$ & $1.85(1.53-2.22)$ \\
\hline Pancreatic & & $3.55(2.23-5.65)$ & & & $1.07(0.68-1.69)$ & $2.36(1.86-3.00)$ \\
\hline Bladder & & $1.66(0.93-2.97)$ & & & $2.07(1.26-3.41)$ & $1.2(0.87-1.70)$ \\
\hline \multicolumn{7}{|l|}{ Cancer stage (vs stage IV) } \\
\hline ॥ & $0.60(0.45-0.80)$ & $0.31(0.22-0.42)$ & & & $0.74(0.54-1.02)$ & $0.52(0.44-0.61)$ \\
\hline III & $0.94(0.73-1.22)$ & $0.33(0.24-0.44)$ & & & $0.71(0.53-0.94)$ & $0.55(0.47-0.63)$ \\
\hline \multicolumn{7}{|l|}{ Histologic grade (vs 9) } \\
\hline 1 & & $0.33(0.17-0.64)$ & & & & $0.54(0.38-0.76)$ \\
\hline 2 & & $0.55(0.39-0.77)$ & & & & $0.71(0.59-0.85)$ \\
\hline 3 & & $0.74(0.54-1.002)$ & & & & $0.87(0.75-1.01)$ \\
\hline 4 & & $1.28(0.71-2.31)$ & & & & $1.02(0.75-1.40)$ \\
\hline \multicolumn{7}{|l|}{ Before index admission } \\
\hline \multicolumn{7}{|l|}{$\geq 1$ SNF stays } \\
\hline \multicolumn{7}{|l|}{ Any therapy } \\
\hline Chemotherapy & & $0.67(0.52-0.87)$ & & & & \\
\hline Radiotherapy & & $1.68(1.28-2.20)$ & & & & $1.18(1.04-1.34)$ \\
\hline Targeted chemotherapy & & & $2.34(1.18-4.62)$ & & & \\
\hline Hypertension & & $0.74(0.55-0.99)$ & & & & $0.82(0.72-0.94)$ \\
\hline \multicolumn{7}{|l|}{ Diabetes } \\
\hline COPD & & & & & & $1.16(1.02-1.32)$ \\
\hline \multicolumn{7}{|l|}{ Heart disease } \\
\hline Stroke & & & $0.47(0.25-0.89)$ & & & \\
\hline Cognitive disorder & $0.44(0.27-0.69)$ & $1.84(1.17-2.88)$ & & & & \\
\hline Alcohol use disorder & & & & & $2.39(1.23-4.63)$ & \\
\hline Depressive disorder & & & $1.60(1.08-2.38)$ & & & \\
\hline
\end{tabular}




\begin{tabular}{|c|c|c|c|c|c|c|}
\hline Variable & $\begin{array}{c}\text { Any Treatment } \\
\text { OR }(95 \% \mathrm{Cl})\end{array}$ & $\begin{array}{c}\text { Death } \\
\text { OR }(95 \% \mathrm{Cl})\end{array}$ & $\begin{array}{c}\text { Hospice } \\
\text { OR }(95 \% \mathrm{Cl})\end{array}$ & $\begin{array}{c}\text { Hospice } \\
\text { LOS <3 D } \\
\text { OR }(95 \% \mathrm{Cl})\end{array}$ & $\begin{array}{c}\text { 30-D } \\
\text { Readmission } \\
\text { OR }(95 \% \mathrm{CI})\end{array}$ & $\begin{array}{l}\text { Months to } \\
\text { Death } \\
\text { HR }(95 \% \mathrm{Cl})\end{array}$ \\
\hline \multicolumn{7}{|l|}{ Index hospital } \\
\hline \multicolumn{7}{|l|}{ Region (vs West) } \\
\hline Northeast & & & $1.98(1.40-2.81)$ & & & \\
\hline Midwest & & & $3.90(2.21-6.88)$ & & & \\
\hline South & & & $1.86(1.20-2.88)$ & & & \\
\hline Location: urban (vs rural) & & & & & $2.01(1.22-3.33)$ & $0.69(0.53-0.91)$ \\
\hline Type: teaching (vs nonteaching) & & $0.76(0.60-0.96)$ & & & & $0.85(0.76-0.96)$ \\
\hline LOS & & & $0.96(0.94-0.99)$ & $0.92(0.86-0.99)$ & $1.03(1.01-1.05)$ & \\
\hline
\end{tabular}

Abbreviations: COPD, chronic obstructive pulmonary disease; HR, hazard ratio; LOS, length of stay; NOS, not otherwise specified; OR, odds ratio; SES, socioeconomic status; SNF, skilled nursing facility.

aGrade 1, well-differentiated/differentiated, NOS; Grade 2, moderately differentiated/moderately well-differentiated/intermediate differentiation; Grade 3, poorly differentiated; Grade 4, undifferentiated/anaplastic; Grade 9, differentiation not determined, not stated, or not applicable.

prior oncologic treatment of any kind. We posit that the functional decline that patients with unestablished cancer experience is primarily driven by the malignancy itself, whereas patients with established cancer and exposure to cancer-directed therapy might experience functional decline as a result of their treatment or a complication of treatment and thus might be more likely to regain functional strength to receive future cancer-directed therapy after an SNF stay.

Results of this study should prompt consideration of the financial implications of discharging patients with cancer with functional decline to an SNF. Although readmissions were not statistically different between the discharge locations, $29 \%$ of patients were readmitted within 6 months of their acute care hospitalization. High hospital readmission rates from the postacute care setting have become exceedingly problematic for hospitals and SNFs, because they now receive financial penalties for these readmissions and lower quality metrics. This is largely being driven by the Medicare Hospital Readmissions Reduction Program and the increasing prevalence of bundled payments and shared-savings programs since the passage of the Patient Protection and Affordable Care Act. $^{21,22}$ Feder et al ${ }^{23}$ studied hospital and SNF clinicians' perceptions of goals-of-care discussions for patients discharged to an SNF and found that discordant goals of care among patients, family members, and clinicians were perceived to contribute to poor patient outcomes at SNFs and to increased hospital readmissions.

Our study has several limitations. The SEER-Medicare dataset is a secondary claims database that does not provide a measure of functional status or social support, which are both factors that might influence disposition after acute care hospitalization and the outcomes we measured. We do not know the goals of patients discharging to an SNF. Regardless, the Centers for Medicare \& Medicaid Services has set up a reimbursement infrastructure with the view that SNFs are to serve a rehabilitative function. Our analysis of SEER-Medicare data from 2010 to 2013 occurred immediately before the increase in immunotherapy use-a treatment that might not have the same PS requirements as traditional cytotoxic chemotherapy. Preliminary research has shown that older patients with an ECOG PS of $\geq 2$ had poor outcomes despite receipt of immunotherapy and that overall survival was primarily driven by a patient's ECOG PS. ${ }^{24}$ Further research is needed to understand the relationship between immunotherapy and functional outcomes, because these patient populations were underrepresented in clinical trials involving immunotherapies. ${ }^{25}$ We note that patients with earlier-stage disease might have received surgery as primary treatment and might not have been candidates for adjuvant treatment. Evaluating postacute care outcomes of patients with cancer undergoing primary surgical treatment is an important line of inquiry but beyond the scope of this article. Finally, future receipt of outpatient oral cancer-directed therapy was not captured in this study because oral medications are covered by Medicare Part D and these claims were not analyzed.

\section{Conclusions}

Our study shows that most patients with stage II-IV colorectal, lung, bladder, and pancreatic cancer discharged to an SNF are less likely to receive cancerdirected treatment and more likely to die compared with those who are functionally able to discharge home. Next steps include better understanding patients' goals of care when discharging to an SNF, and developing and 
implementing targeted interventions that improve palliative care delivery models to patients in the SNF setting.

Submitted July 23, 2019; accepted for publication January 10, 2020.

Disclosures: The authors have disclosed that they have not received any financial consideration from any person or organization to support the preparation, analysis, results, or discussion of this article.

Funding: This project is supported by the Palliative Care Research Cooperative Group funded by the National Institute of Nursing Research (U24NR014637) and the Population Health Shared Resources, University of Colorado Cancer Center (P30CA046934).

Correspondence: Sarguni Singh, MD, University of Colorado Denver, Division of Hospital Medicine, Leprino Building, 4th Floor, 12401 East 17th Avenue, Mailstop F-782, Aurora, CO 80045. Email: sarguni.singh@ucdenver.edu

\section{References}

1. Verweij NM, Schiphorst AH, PronkA, et al. Physical performance measures for predicting outcome in cancer patients: a systematic review. Acta Oncol 2016;55:1386-1391.

2. Studenski S, Perera S, Patel K, et al. Gait speed and survival in older adults. JAMA 2011;305:50-58.

3. Stanaway FF, Gnjidic D, Blyth FM, et al. How fast does the Grim Reaper walk? Receiver operating characteristics curve analysis in healthy men aged 70 and over. BMJ 2011;343:d7679.

4. Cesari M, Kritchevsky SB, Newman AB, et al. Added value of physical performance measures in predicting adverse health-related events: results from the Health, Aging And Body Composition Study. J Am Geriatr Soc 2009; $57: 251-259$

5. Burke RE, Juarez-Colunga E, Levy C, et al. Rise of post-acute care facilities as a discharge destination of US hospitalizations. JAMA Intern Med 2015; 175:295-296.

6. Mor $\mathrm{V}$, Intrator $\mathrm{O}$, Feng $\mathrm{Z}$, et al. The revolving door of rehospitalization from skilled nursing facilities. Health Aff (Millwood) 2010;29:57-64.

7. Neuman, MD, Wirtalla C, Werner RM. Association between skilled nursing facility quality indicators and hospital readmissions. JAMA 2014; 312:1542-1551.

8. Werner RM, Konetzka RT. Trends in post-acute care use among Medicare beneficiaries: 2000 to 2015. JAMA 2018;319:1616-1617.

9. Redberg RF. The role of post-acute care in variation in the Medicare program. JAMA Intern Med 2015;175:1058.

10. Lester PE, Stefanacci RG, Feuerman M. Prevalence and description of palliative care in US nursing homes: a descriptive study. Am J Hosp Palliat Care 2016:33:171-177.

11. National Cancer Institute, Surveillance, Epidemiology, and End Results (SEER) Program. Overview of the SEER Program. Accessed October 1, 2019. Available at: https://seer.cancer.gov/about/overview.html

12. National Cancer Institute. SEER-Medicare: Medicare Enrollment \& Claims Data. Accessed October 1, 2019. Available at: https://healthcaredelivery. cancer.gov/seermedicare/medicare/

13. National Cancer Institute. SEER-Medicare: How the SEER \& Medicare Data are Linked. Accessed October 1, 2019. Available at: https:// healthcaredelivery.cancer.gov/seermedicare/overview/linked.html
14. Center for Medicare and Medicaid Services. Chronic Conditions Data Warehouse. Accessed October 1, 2019. Available at: https://www. ccwdata.org/web/guest/condition-categories

15. Eastern Cooperative Oncology Group. ECOG performance status. Accessed October 1, 2019. Available at: http://ecog-acrin.org/resources/ ecog-performance-status.

16. Schnipper LE, Smith TJ, Raghavan D, et al. American Society of Clinical Oncology identifies five key opportunities to improve care and reduce costs: the top five list for oncology. J Clin Oncol 2012;30: 1715-1724

17. Singh $S$, Cortez D, Maynard D, et al. Characterizing the nature of scan results discussions: insights into why patients misunderstand their prognosis. J Oncol Pract 2017;13:e231-239.

18. Martoni AA, Tanneberger S, Mutri V. Cancer chemotherapy near the end of life: the time has come to set guidelines for its appropriate use. Tumor 2007; $93: 417-422$

19. Teno JM, Gozalo P, Trivedi AN, et al. Site of death, place of care, and health care transitions among US Medicare beneficiaries, 2000-2015. JAMA 2018;320:264-271.

20. US Centers for Medicare \& Medicaid Services. Medicare Care Choices Model enables concurrent palliative and curative care. J Pain Palliat Care Pharmacother 2015;29:401-403.

21. Berenson RA, Paulus RA, Kalman NS. Medicare's readmissions-reduction program—a positive alternative. N Engl J Med 2012;366:1364-1366

22. Cutler DM, Ghosh K. The potential for cost savings through bundled episode payments. N Engl J Med 2012;366:1075-1077.

23. Feder SL, Britton MC, Chaudhry SI. "They need to have an understanding of why they're coming here and what the outcomes might be." Clinician perspectives on goals of care for patients discharged from hospitals to skilled nursing facilities. J Pain Symptom Manage 2018;55:930-937.

24. Muchnik E, Loh KP, Strawderman M, et al. Immune checkpoint inhibitors in real-world treatment of older adults with non-small cell lung cancer. J Am Geriatr Soc 2019;67:905-912.

25. Kanesvaran R, Cordoba R, Maggiore R. Immunotherapy in older adults with advanced cancers: implications for clinical decision-making and future research. Am Soc Clin Oncol Educ Book 2018;38:400-414. 
Supplemental online content for:

\section{Outcomes of Patients With Cancer Discharged to a Skilled Nursing Facility After Acute Care Hospitalization}

Sarguni Singh, MD; Megan Eguchi, MPH; Sung-Joon Min, PhD; and Stacy Fischer, MD

J Natl Compr Canc Netw 2020;18(7):856-865

eAppendix 1: Codes for Treatment Variables 


\section{eAppendix 1. Codes for Treatment Variables}

\begin{tabular}{|c|c|c|c|c|c|}
\hline Variable & ICD-9 Diagnosis Codes & ICD-9 Procedure Codes & HCPCS codes & Revenue Codes & NDC Codes \\
\hline Radiotherapy & V58.0 & $\begin{array}{l}92.21-92.29, \\
92.30-92.39,92.41\end{array}$ & $\begin{array}{l}76370,76950,77014, \\
77261-77263,77280,77285, \\
77290,77295,77299-77301, \\
77305,77310,77315,77321, \\
77326-77328,77331-77334, \\
77336,77338,77370-77373, \\
77399,77401-77499,77520, \\
77522,77523,77525, \\
77750-77799,0073 T, 0082 T, \\
\text { 0083T, 0182T, 0520F, G0173, } \\
\text { G0174, G0178, G0242, } \\
\text { G0243, G0251, G0256, } \\
\text { G0261, G0273, G0274, } \\
\text { G0338-G0340 }\end{array}$ & $\begin{array}{l}0330,0333,0339 \\
0342,0344\end{array}$ & \\
\hline Chemotherapy & $\begin{array}{l}\text { V58.1, V58.11, } \\
\text { V58.12 }\end{array}$ & $00.10,99.25,99.28$ & $\begin{array}{l}\text { 96400-96599, } \\
\text { C8953-C8955, C9127, } \\
\text { C9205, C9213, C9215, } \\
\text { C9235, C9257, C9414, } \\
\text { C9418, C9425, C9427, } \\
\text { C9431, C9432, C9440, } \\
\text { J8510, J8520, J8521, J8530, } \\
\text { J8600, J8610, J8700, J8705, } \\
\text { J8999-J9999, } \\
\text { Q0083-Q0085, S0177, } \\
\text { S9329-S9331, 51720, 61517, } \\
\text { 0519F, S0116, Q2024, } \\
\text { C9025, C9296, J7527 }\end{array}$ & & \\
\hline Bevacizumab & & & C9257, J9035, S0116, Q2024 & & 50242006001,50242006101 \\
\hline Cetuximab & & & J9055 & & 66733094823,66733095823 \\
\hline Everolimus & & & J7527 & & $\begin{array}{l}00078056651,00078056751, \\
00078059451,00078062051, \\
00078062651,00078062751, \\
00078062851\end{array}$ \\
\hline Panitumumab & & & $J 9303$ & & $\begin{array}{l}55513095401,55513095501, \\
55513095601,59703095601\end{array}$ \\
\hline Ramucirumab & & & C9025, J9308 & & 00002766901,00002767801 \\
\hline Ziv-aflibercept & & & C9296, J9400 & & $\begin{array}{l}00024584001,00024584003, \\
00024584101\end{array}$ \\
\hline
\end{tabular}

HCFA specialty codes for any oncology visit: 83, hematology/oncology; 90, medical oncology; 91, surgical oncology; 92, radiation oncology; 98, gynecologist/ oncologist.

Abbreviations: HCFA, Health Care Finance Administration; HCPCS, Healthcare Common Procedure Coding System; NDC, National Drug Code. 